\title{
Studying the Kidney Textural Using Statistical Features and Local Binary Pattern
}

\author{
Alyaa Hussein Ali \\ College of Science for Women, Baghdad University, Baghdad-Iraq.
}

\begin{abstract}
The structural of image either medical or any other images is describe by their texture, thus the texture is consider as the characteristic information of the image. In this search the Computed tomography (CT) images is used, the images size are $512 \times 512$ of (12) images for different kidney cases, four for Cyst, four for Fibrosis and four for Stone case. The watershed segmentation used to segment the Cyst, Fibrosis and Stone from the healthy texture as well as, the "Local Binary Pattern" (LBP) which is a texture descriptor used for locating the Cyst, Fibrosis and stone from the rest images, finally the textural spectrum is used as statistical features, more over the geometrical features are calculated in order to describe the shape and geometry of Cyst, Fibrosis and Stone of the Kidney by finding the irregularity value. The texture features accuracy which are obtained in this search are, for the Fibrosis, the Local Binary Pattern is $89.91 \%$, the Textural Spectrum 92.65\% and Local Binary with the Textural Spectrum is $94.55 \%$. For the Cyst texture features accuracy are, the Local Binary Pattern is $93.65 \%$, the Textural Spectrum $94.56 \%$ and Local Binary with the Textural Spectrum is $96 \%$. And for the Stone the texture features accuracy are, the Local Binary Pattern is $88.4 \%$, the Textural Spectrum $91.36 \%$ and Local Binary with the Textural Spectrum is 95\%. [DOI: 10.22401/JUNS.20.4.11]
\end{abstract}

Keywords: Local Binary Pattern, Watershed segmentation, Geometrical Features, Statistical Features.

\section{Introduction}

A lot of information about the inside of human body can be study by using the medical image processing. It can be interpreted by the physician depending on the type of the images which are used. The Computed tomography (CT) images give a huge description about the kidney texture and it include a lot of information about the construction of the images. The watershed segmentation in which morphological operation used in segmented the images, (Beucher and Lantuejoul) were the first which use the watershed concept [1]. The method which are adopted in this search based on two steps, the first one is detecting the local changes in the image by boundary based. The second step depend on measuring the similarities between the pixel regions [2]. The "texture analysis" is the most important procedure to extracted the information features from the images, (Tuceryan and Jain) it is used the statistical and geometrical features to describe the textural information. The approaches to classifying the texture depend on either region based or pixel based, for the region based the image must segmented to homogenous textural region while, for the pixel based the spectral information is used to classify the pixel in the images[3]. Consider the uniform pattern of the images as a local features. The Local Binary Pattern (LPB) and textural spectrum (TS) are used as an extracted features methods [4], the "texture spectrum" is used as a filtering process by (He and Wang, 1991) the texture spectrum based on extraction the local texture information for images pixels from the image spectrum. This paper describe the work as follows, first group of CT kidney images are taken. The kidney extracted from the rest images, a watershed segmentation is applied to located the Fibrosis, Cyst and Stone in the CT images, the Local Binary Pattern with Textural spectrum are applied on the region of interest, the geometrical and statistical features are calculated to describe the Fibrosis, Cyst and Stone shape. In the end, the feature texture accuracy is computed to give a description about the kidney diseases.

\section{Watershed}

The watershed segmentation is a process of image classification, it is first used by Beucher [1]. This segmentation based on the pixels classifier regions using the gradient 
value of an image. Features and analyzing the weak points along the boundaries region, the principles for this segmentation follows the steps:

1. Marking the local minima of the gradient of an image. This leads to over the segment of the image together, to overcome this problem, a method has been proposed by Meyer and Beucher [5]. It is called markercontrolled segmentation.

2. Use the specific marker position which have been determined either by morphological operators or by the user. First morphological tools used in segmentation: Each object in the image will define with a certain properties which mark the objects and they are named object markers. The same procedure will be done for the background where there is no pixel belong to the objects and it is called background markers. A gradient image is applied to get specific contours for the area of the markers, the final contours search on the gradient image is done by performing the "watershed segmentation process", the gradient is important in making watershed segmentation, since it depend on the homogeneity of the gray level values of each objects in the image. If the segmentation depend on the shape of the object, the distance is needed to perform the segmentation. The need for the distance function and the gradient function are called the morphological tools. Second discussing the over-segmentation between the objects, this can efficiently solved by defining different segmentations level [6].

\section{Methodology}

Three diseases are taken for this search, each diseases four cases which are taken from the Al-kadymaa Hospital, a binary mask image is created to separate the tumor from the rest images and the density slicing technique is used to color the images in order to prepare it for segmentation. The segmentation process is used to separate the defect from the rest images, The region of interest of the kidney is obtained by using mask of white and black value to extracted the kidney from the CT images, the watershed segmentation is applied to extracted the cyst, fibrosis and stone from the image, also the LBP is used to compute the local feature of the image, then apply the Texture spectrum to calculate the Texture Unit (TU) number. The statistical and geometrical features also, obtained to describe the texture properties and shapes. The statistical features which are stander deviation, mean and local homogeny can be obtained as follows:

1. Mean Value: It represent the mean value of the gray levels in the cyst, fibrosis and stone images. The value of the mean is high when the sum of gray level is large $[7,8]$.

Mean $=\sum \frac{\text { Value of the pixel in the image }}{\text { Number of pixel in the image }}$

2. Standard Deviation: The standard deviation (SD, also represented by the Greek letter sigma $\sigma$ ) represent the amount of variation or the dispersion data set can be represented by standard deviation, the low standard deviation refer to the value of data which tend to be close to the mean, while a high standard deviation indicates that the data set is dispersion over a wider range of the data values The SD is calculated for the cyst, fibrosis and stone images as follows [7].

$$
\boldsymbol{\sigma}^{2}{ }_{x, y}=\left[\sum\left(\mathrm{xi}-\boldsymbol{\mu}_{x, y}\right) 2\right] / \mathrm{N}
$$

where $\mathrm{N}$ is the number of pixel in the image, $\mathrm{Xi}$ is the pixel value and $\boldsymbol{\mu}_{\boldsymbol{x}, \boldsymbol{y}}$ is the mean value [7].

3. Local Homogeneity: It represent the measure of smoothness degree, if (LHOM) be the value of the pixel located at $(x, y)$ the equation can be defined as [9].

$$
\mathrm{LHOM}=\frac{\sqrt{\sigma_{x, y}}}{\mu_{x, y}} \frac{\sigma_{x, y}}{}
$$

Where $\boldsymbol{\mu}_{\boldsymbol{x}, \boldsymbol{y}}$ is the local mean and $\boldsymbol{\sigma}_{\boldsymbol{x}, \boldsymbol{y}}$ is the local variance of a pixel located at $(\mathrm{x}, \mathrm{y})$ with block size of $\mathrm{N} \times \mathrm{N}[9]$.

\section{Local Binary Pattern Operator}

(LBP) is the features for texture description. it describe the formation of small patterns in which "LBP" represents the first-order circular derivative pattern of images, the small pattern resulting by series of binary gradient directions. When the first-order pattern fails to extract detailed included in the input object. 
The high-order local pattern operator is needed. Second order which is the "local directional pattern LDP" which can take the change of derivative directions among local neighbors, and encode the turning point in a given direction. The LBP thresholding the neighborhood of each pixel in the image with the value of the center in a given window of $3 \times 3[3]$.

$$
\begin{aligned}
& L B P=\sum_{i=1}^{8} E_{i} 2^{i-1} \\
& E_{i}=\left\{\begin{array}{l}
1 \text { if } \quad V_{i} \geq V_{0} \\
0 \text { if } \quad V_{i}<V_{0}
\end{array}\right.
\end{aligned}
$$

\section{Texture Spectrum}

In $3 \times 3$ window, each pixel is surrounded by 8 neighboring pixels. The local texture information in the square image for the eight pixel can be obtained from a neighborhood of the data pixels, the "texture unit TU" can be extracted by the set accounting eight elements as follows [3]

$$
\begin{aligned}
& \mathrm{TU}=\{\mathrm{E} 1, \mathrm{E} 2, \ldots . \mathrm{E} 8\} . \\
& \boldsymbol{E}_{\boldsymbol{i}}=\left\{\begin{array}{l}
\mathbf{0} \text { if } \boldsymbol{V}_{\boldsymbol{i}}<\boldsymbol{V}_{\mathbf{0}} \\
\mathbf{1} \text { if } \boldsymbol{V}_{\boldsymbol{i}}=\boldsymbol{V}_{\mathbf{0}} \\
\mathbf{2} \text { if } \boldsymbol{V}_{\boldsymbol{i}}>\boldsymbol{V}_{\mathbf{0}}
\end{array}\right.
\end{aligned}
$$

where $\mathrm{E}(\mathrm{i}=1,2, \ldots . .8$,

and $\mathrm{i}=1,2 \ldots 8$ and the element $\mathrm{Ei}$, can have the same position of pixel $i$. when the element of textural unit has one of three possible values. The texture unit number can be calculated by using the following formula:

$$
\mathbf{N}_{\mathrm{TU}}=\sum_{\mathrm{i}=\mathbf{1}}^{8} \mathrm{E}_{\mathrm{i}} \mathbf{3}^{\mathrm{i}-\mathbf{1}}
$$

The percentage of the correct classification can be calculated as follows [10].

$\frac{\text { number of sub image correctly classified }}{\text { number of sub image consider for testing }} \times \mathbf{1 0 0}$.

\section{Geometrical Features}

The geometrical features can be describe by determining the area, perimeter and irregularity index. The area is finding by summing all the white pixels in the image matrix which has one value, while the background which has zero value is ignored. The irregularity index is computed by the following equation

$$
I=\frac{4 \pi A}{p^{2}}
$$

$A=\sum$ White pixel in the image

Where, $\mathrm{p}$ is the perimeter represented by the bounder of the Fibrosis, Cyst and Stone, A is the area of Fibrosis, Cyst and Stone in the kidney images. The irregularity index is equal to 1 only for circle and it is $<1$ for any other shape [11].

\section{The Study Cases}

Cysts cases: Figs. (1),(2),(3),(4) represent the cysts cases each of the right and left side of the kidney have cysts, the cysts are nearly regular in shape. Tables (1),(2),(3),(4) shows the statistical features for each side of the kidney. Fig.(5) shows the LBP for cyst case, Table $(13,14)$ represent the geometrical features for the cyst, table (21) shows the textural feature accuracy for cyst case.

\section{Kidney Failure Cases (kidney Fibrosis):}

Fig.(6),(7),(8),(9) shows the fibrosis cases each side left and right have fibrosis, Tables (5),(6),(7),(8) shows the statistical features for each side of the kidney. Fig.(10) shows the LBP for fibrosis case, Table $(15,16)$ represent the geometrical features for the fibrosis, Table (21) shows the textural feature accuracy for fibrosis case.

Stone Cases: Fig. $(11,12,13,14)$ shows the stone cases each side left and right have stone expect image No.1 and image No.4 only the right side have stone, Tables (9),(10),(11),(12) shows the statistical features for each side of the kidney. Fig.(15) shows the LBP for fibrosis case, Table $(17,18)$ represent the geometrical features, Table (21) shows the textural feature accuracy for stone case.

\section{Discussion}

A statistical feature are calculated for the cyst, fibrosis and the stone parts, the Local Binary Pattern which is a textural features extraction are calculated for these cases, the images have been derived to the third derivation which is the best result obtained in calculating the LBP. The texture spectrum are also calculated to improve the textural unit. The geometrical feature represented by the area, circumference and the irregularity value represented the shape of the detected tumor. This value helps the physician to get the exact 
size and shape of tumor to give the patient the suitable amount of treatment Tables $(13,14$, $15,16,17,18)$ shows the irregularity values, table (19) represented the average value for the three cases of the statistical features, Table (20) is the average value of the irregularity for the three cases, Table (21) is the textural feature accuracy for the three cases. The cyst has the greatest value in the statistical features and the most regular between the stone and fibrosis cases. The value of stander deviation is higher than the other cases (i-e) the variance is high and the dispersion around the mean is also high, the local homogeneity and mean for the cyst is high than the other cases. This can clearly see in Fig.(16).
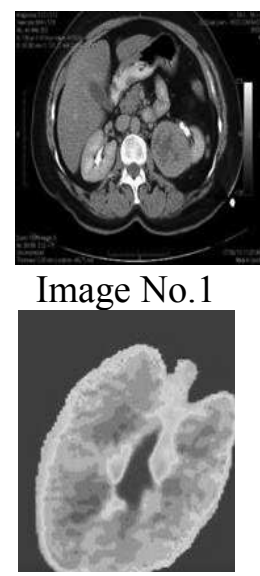

Watershed Segmentation

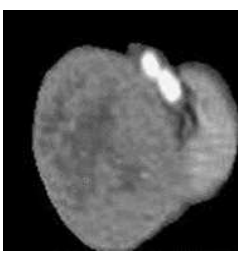

Left kidney

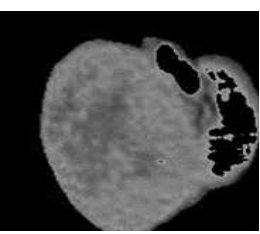

Unhealthy

Tissues

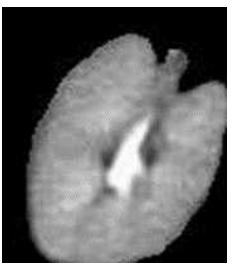

Right kidney

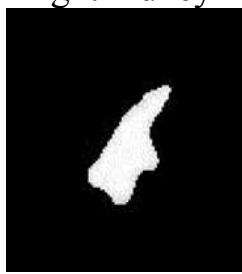

Cyst

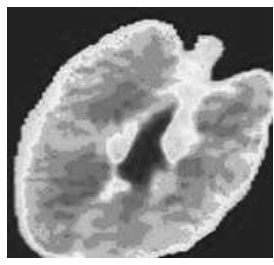

Coloring Image

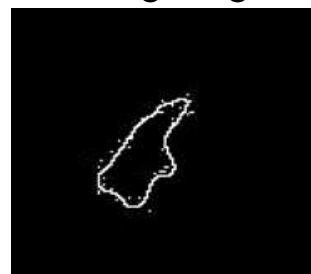

Boundary of Cyst

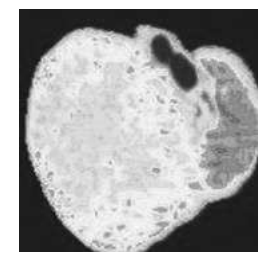

Coloring Image

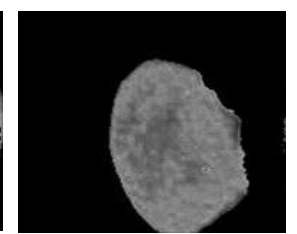

Cyst

Boundary of

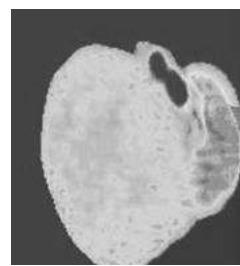

Watershed Segmentation

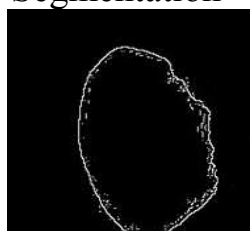
Cyst
Fig.(1): Shows the watershed segmentation with cyst case for Image No.1.
Table (1)

The statistical features for right and left kidney for cyst case.

\begin{tabular}{|c||c||c||c|}
\hline $\begin{array}{c}\text { Image } \\
\text { No.1 }\end{array}$ & $\begin{array}{c}\text { Standard } \\
\text { Deviation }\end{array}$ & Mean & $\begin{array}{c}\text { Local } \\
\text { Homogeneity }\end{array}$ \\
\hline \hline $\begin{array}{c}\text { Right } \\
\text { kidney }\end{array}$ & 53.8721 & 130.7107 & 0.9865 \\
\hline $\begin{array}{c}\text { Left } \\
\text { kidney }\end{array}$ & 59.0117 & 148.4303 & 0.9900 \\
\hline
\end{tabular}

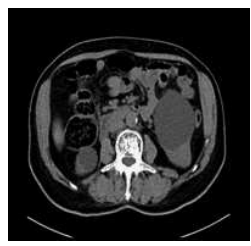

Image No.2

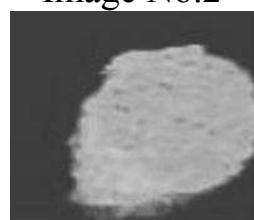

Watershed segmentation

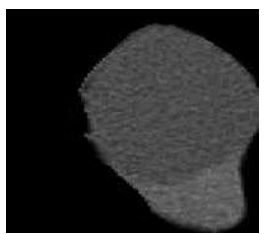

Left kidney

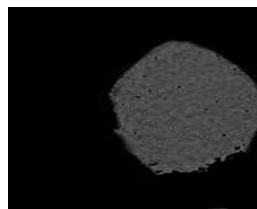

Cyst

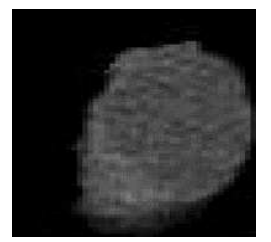

Right kidney

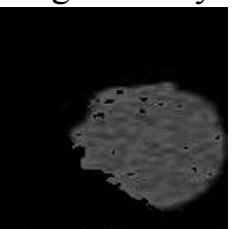

Cyst

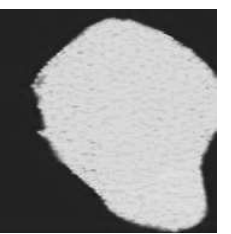

Coloring Image

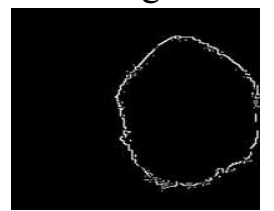

Boundary of Cyst

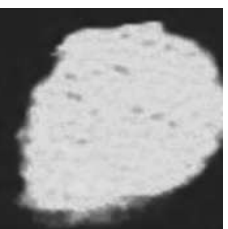

After coloring

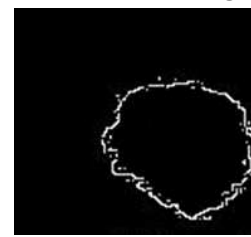

Boundary of Cyst

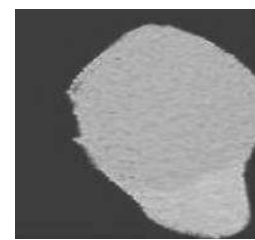

Watershed segmentation
Fig.(2): Shows the watershed segmentation with cyst case for Image No.2.

Table (2)

The statistical features for right and left kidney for cyst case.

\begin{tabular}{||c|c|c|c||}
\hline $\begin{array}{c}\text { Image } \\
\text { No.2 }\end{array}$ & $\begin{array}{c}\text { Standard } \\
\text { Deviation }\end{array}$ & Mean & $\begin{array}{c}\text { Local } \\
\text { Homogeneity }\end{array}$ \\
\hline \hline $\begin{array}{c}\text { Right } \\
\text { kidney }\end{array}$ & 54.8313 & 122.3128 & 0.9981 \\
\hline $\begin{array}{c}\text { Left } \\
\text { kidney }\end{array}$ & 56.9177 & 129.0382 & 0.9978 \\
\hline
\end{tabular}




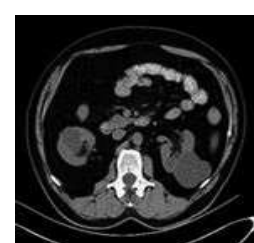

Image No.3

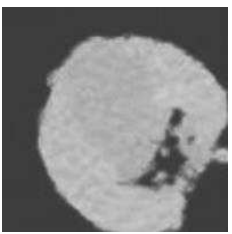

Watershed

Segmentation

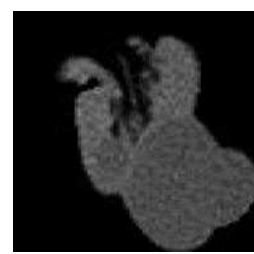

Left kidney

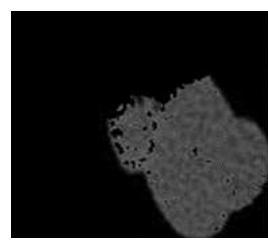

Cyst

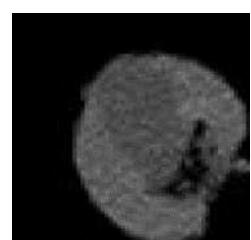

Right kidney

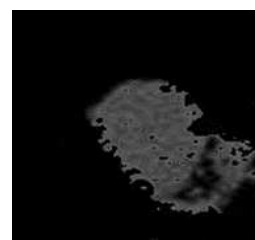

Cyst

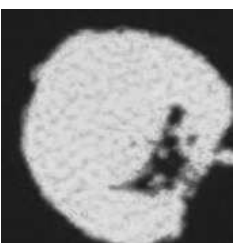

Coloring

Image

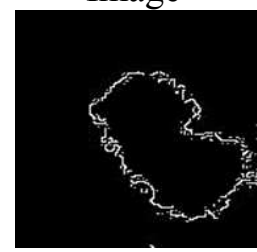

Boundary of Cyst

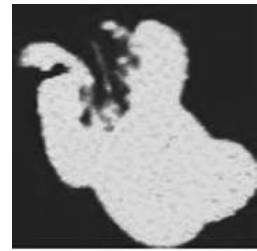

Coloring Image

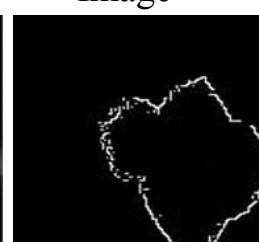

Boundary of Cyst

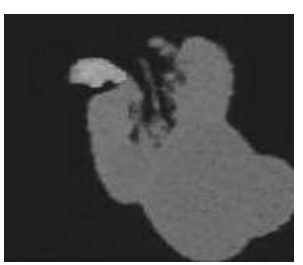

Watershed Segmentation

Fig.(3): Shows the watershed segmentation with cyst case for Image No.3.

Table (3)

The statistical features for right and left kidney for cyst case.

\begin{tabular}{|c||c|c||c||}
\hline $\begin{array}{c}\text { Image } \\
\text { No.3 }\end{array}$ & $\begin{array}{c}\text { Standard } \\
\text { Deviation }\end{array}$ & Mean & $\begin{array}{c}\text { Local } \\
\text { Homogeneity }\end{array}$ \\
\hline \hline $\begin{array}{c}\text { Right } \\
\text { kidney }\end{array}$ & 56.9897 & 131.4551 & 0.9966 \\
\hline $\begin{array}{c}\text { Left } \\
\text { kidney }\end{array}$ & 58.8534 & 160.7228 & 0.9966 \\
\hline
\end{tabular}
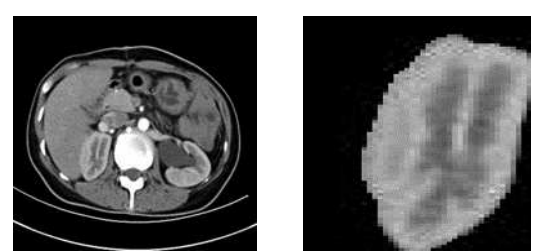

Right kidney

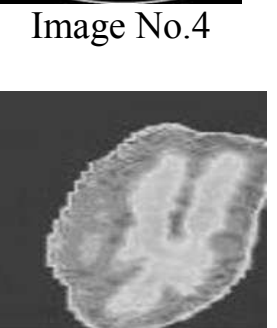

Watershed

Segmentation

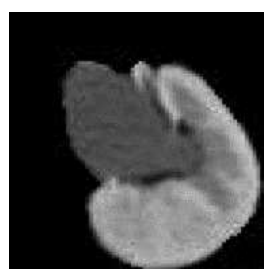

Left kidney

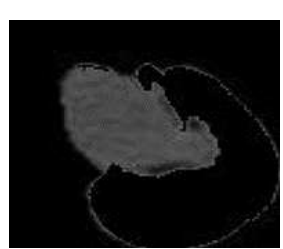

Unhealthy tissues

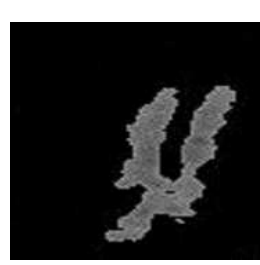

Cyst

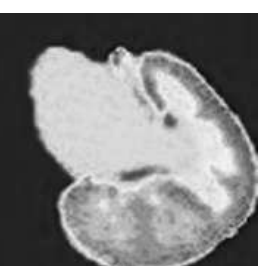

Coloring

Image

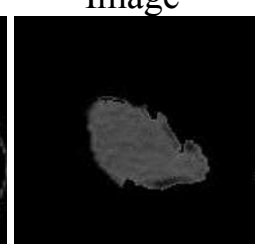

Cyst

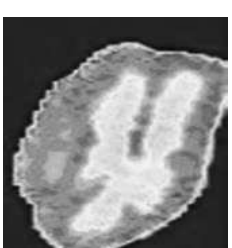

Coloring

Image

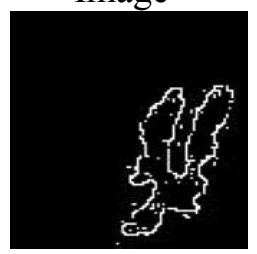

Boundary of Cyst

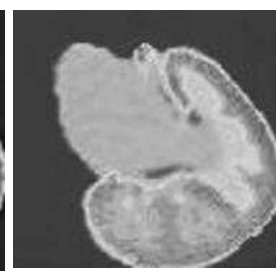

Watershed Segmentation

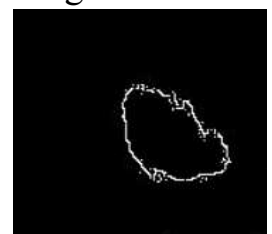

Boundary of cyst

Fig.(4): Shows the watershed segmentation with cyst case for Image No.4.

Table (4)

The statistical features for right and left kidney for cyst case.

\begin{tabular}{||c||c|c||c||}
\hline $\begin{array}{c}\text { Image } \\
\text { No.4 }\end{array}$ & $\begin{array}{c}\text { Standard } \\
\text { Deviation }\end{array}$ & Mean & $\begin{array}{c}\text { Local } \\
\text { Homogeneity }\end{array}$ \\
\cline { 1 - 4 } $\begin{array}{c}\text { Right } \\
\text { Kidney }\end{array}$ & 45.4338 & 89.729 & 0.9956 \\
\hline \hline $\begin{array}{c}\text { Left } \\
\text { Kidney }\end{array}$ & 55.0138 & 131.0059 & 0.9959 \\
\hline
\end{tabular}




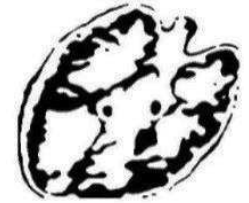

Image No1.right side

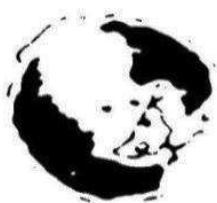

Image No. 3 right side

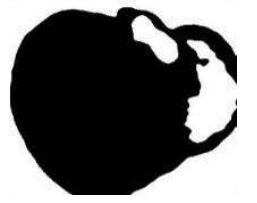

Image No.1 left side

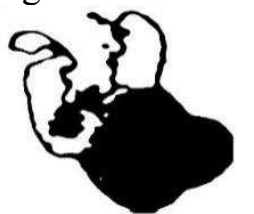

Image No.3 left side

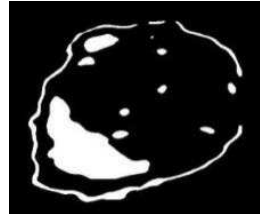

Image No. 2 right side

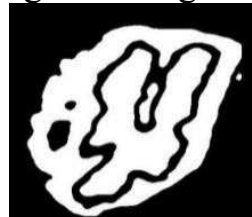

Image No.4 right side

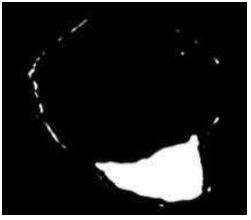

Image No.2 left side

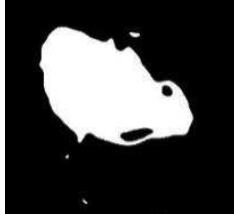

Image No.4 left side

Fig.(5): Shows the LBP Images for cysts cases.

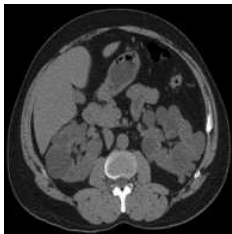

Image No.1

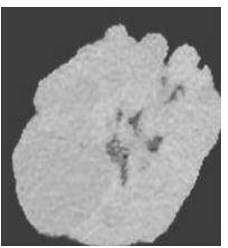

Watershed

Segmentation

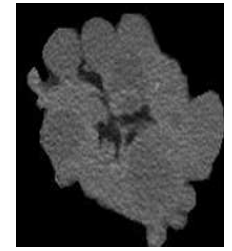

Left Kidney

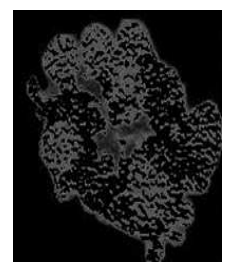

Fibrosis

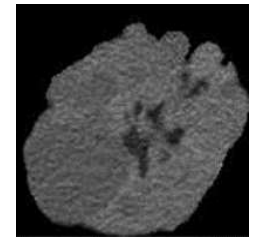

Right kidney

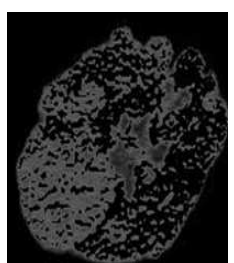

Fibrosis

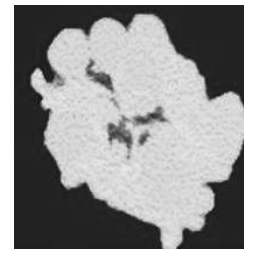

Coloring Image

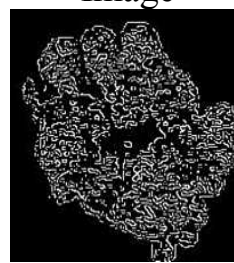

Boundary of Fibrosis

Fig.(6): Shows the watershed segmentation with fibrosis case for Image No.1.

Table (5)

The statistical features for right and left kidney for fibrosis case.

\begin{tabular}{|c||c||c|c||}
\hline Image No.1 & $\begin{array}{c}\text { Standard } \\
\text { Deviation }\end{array}$ & Mean & $\begin{array}{c}\text { Local } \\
\text { Homogeneity }\end{array}$ \\
\hline \hline Right kidney & 41.2874 & 84.2586 & 0.9766 \\
\hline \hline Left Kidney & 21.8406 & 59.5759 & 0.9715 \\
\hline
\end{tabular}

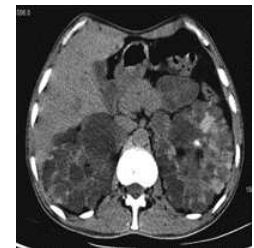

Image No.2

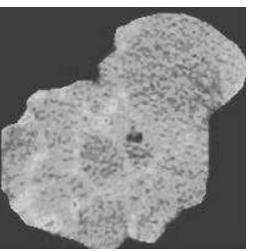

Watershed segmentation

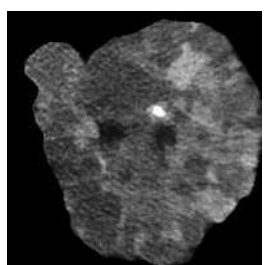

Left kidney

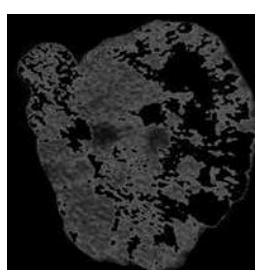

Fibrosis

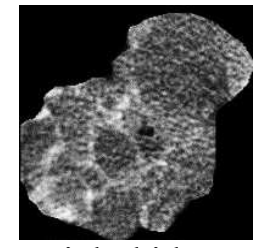

Right kidney

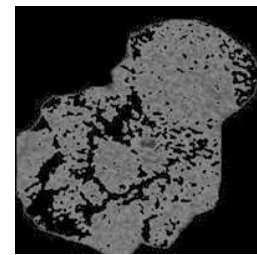

Fibrosis

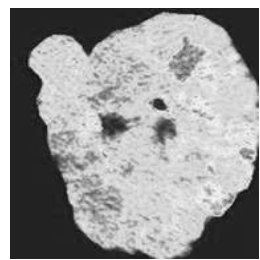

Coloring Image

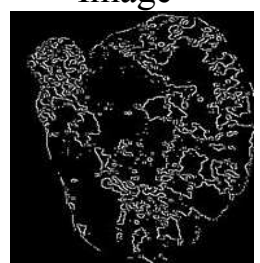

Boundary of Fibrosis

Fig.(7): Shows the watershed segmentation with fibrosis case for Image No.2.

Table (6)

The statistical features for right and left kidney for fibrosis case.

\begin{tabular}{||c||c|c||c||}
\hline Image No.2 & $\begin{array}{c}\text { Standard } \\
\text { Deviation }\end{array}$ & Mean & $\begin{array}{c}\text { Local } \\
\text { Homogeneity }\end{array}$ \\
\hline \hline Right Kidney & 45.8182 & 93.3369 & 0.9644 \\
\hline \hline Left Kidney & 41.6079 & 85.7364 & 0.9770 \\
\hline
\end{tabular}




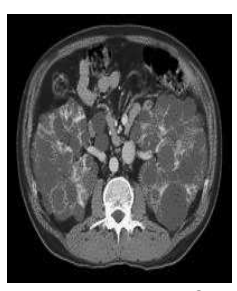

Image No.3

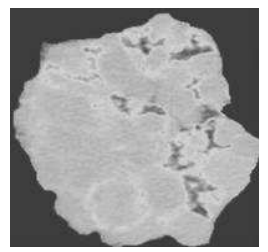

Watershed segmentation

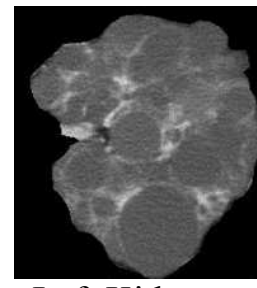

Left Kidney

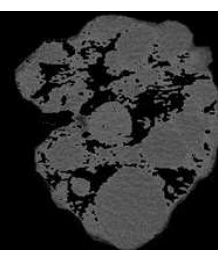

Fibrosis

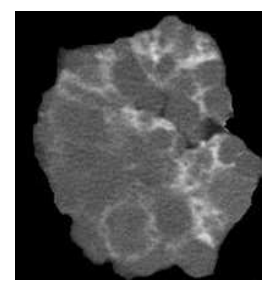

Right kidney

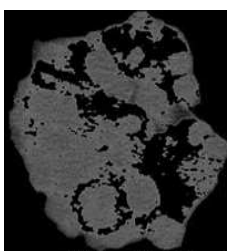

Fibrosis

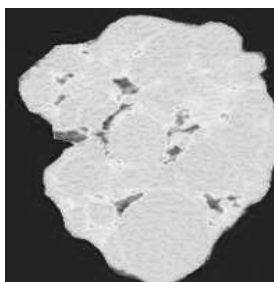

Coloring Image

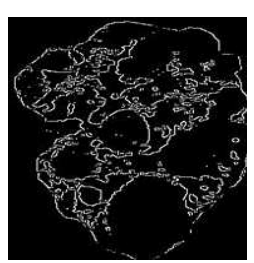

Boundary of fibrosis

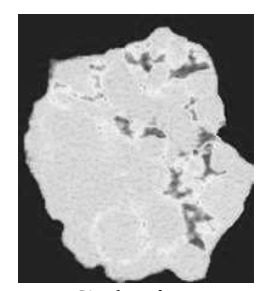

Coloring

Image

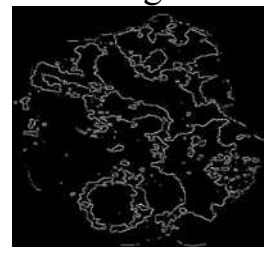

Boundary of

fibrosis

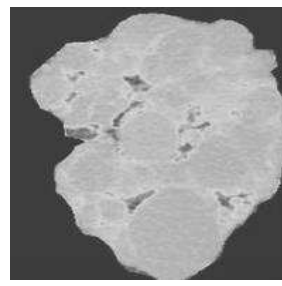

Watershed

segmentation

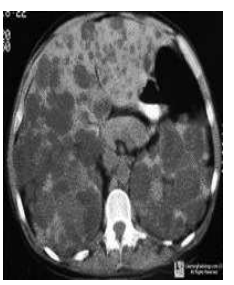

Image No.4

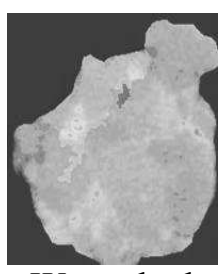

Watershed segmentation

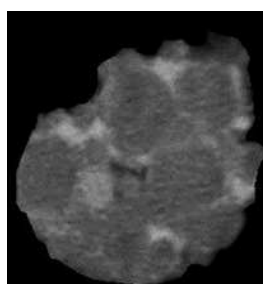

Left kidney

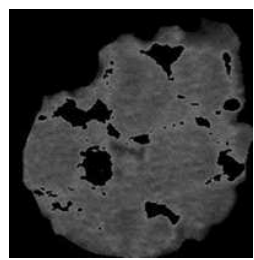

Fibrosis

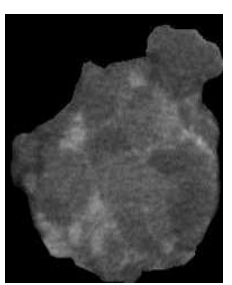

Right kidney

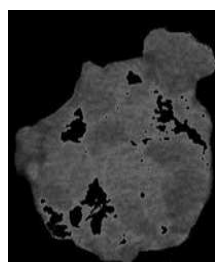

Fibrosis

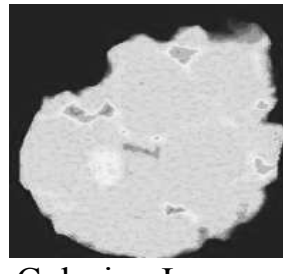

Coloring Image

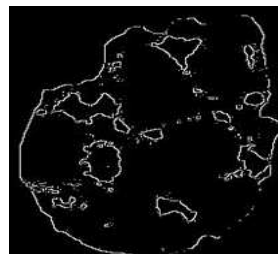

Boundary of Fibrosis

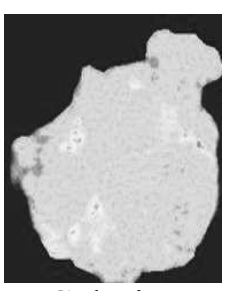

Coloring Image

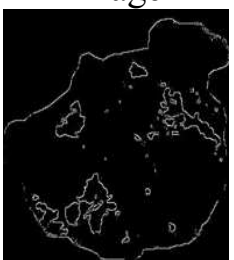

Boundary of Fibrosis

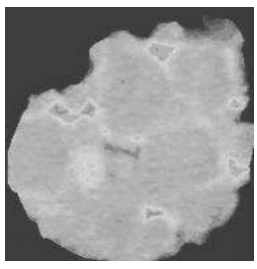

Watershed segmentation

Fig.(8): Shows the watershed segmentation with fibrosis case for Image No3.

Table (7)

The statistical features for right and left kidney for fibrosis case.

\begin{tabular}{||c||c||c||c|}
\hline Image No.3 & $\begin{array}{c}\text { Standard } \\
\text { Deviation }\end{array}$ & Mean & $\begin{array}{c}\text { Local } \\
\text { Homogeneity }\end{array}$ \\
\hline \hline Right Kidney & 45.0196 & 89.642 & 0.9644 \\
\hline Left Kidney & 47.7269 & 90.6058 & 0.9724 \\
\hline
\end{tabular}

Fig.(9): Shows the watershed segmentation with fibrosis case for Image No4.

\section{Table (8)}

The statistical features for right and left kidney for fibrosis case.

\begin{tabular}{||c||c||c||c||}
\hline Image No.4 & $\begin{array}{c}\text { Standard } \\
\text { Deviation }\end{array}$ & Mean & $\begin{array}{c}\text { Local } \\
\text { Homogeneity }\end{array}$ \\
\hline \hline Right Kidney & 44.1633 & 87.8884 & 0.9806 \\
\hline \hline Left Kidney & 45.4424 & 90.7073 & 0.9908 \\
\hline
\end{tabular}




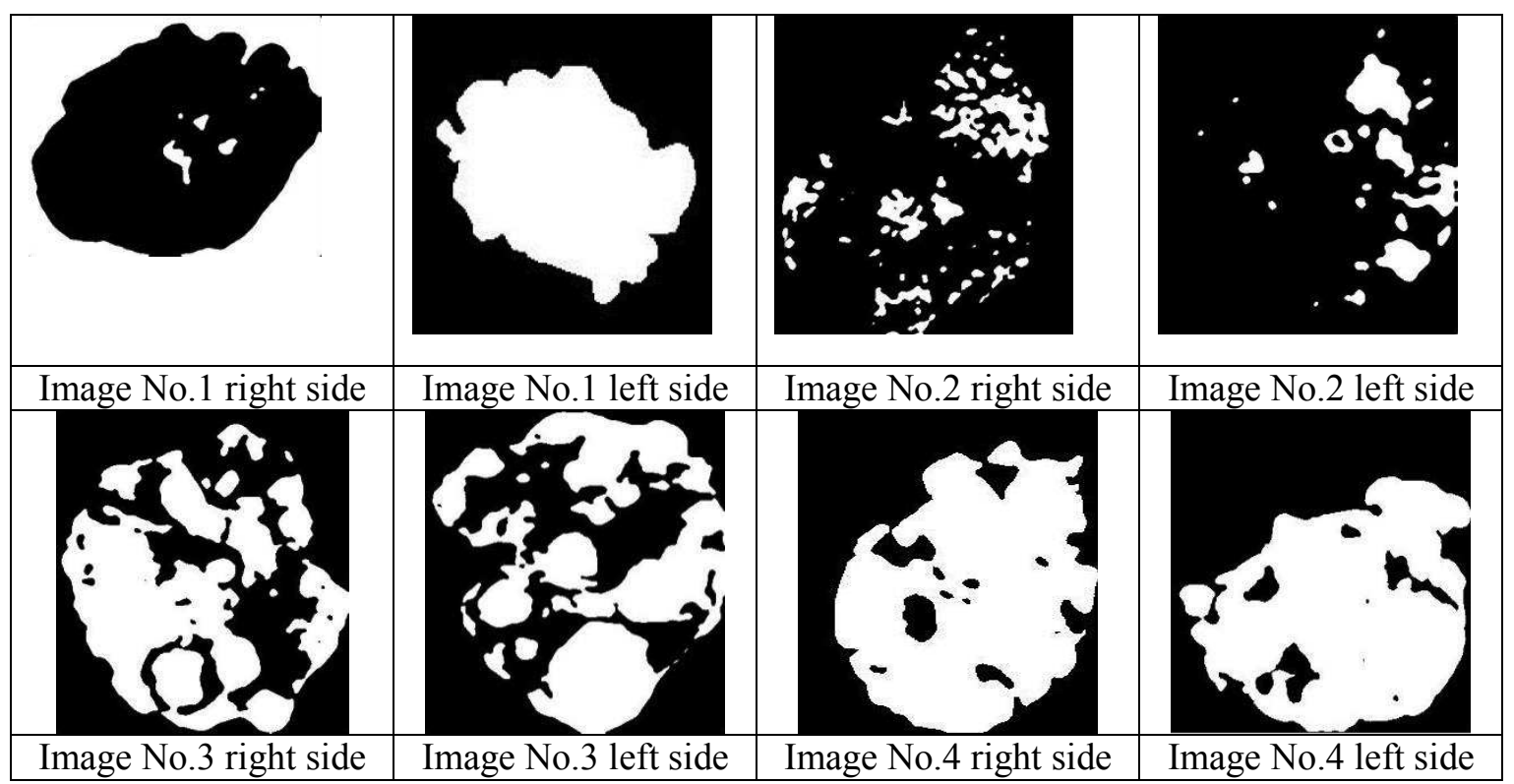

Fig.(10): Shows the LBP Images for fibrosis cases.

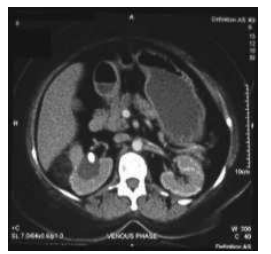

Image No.1

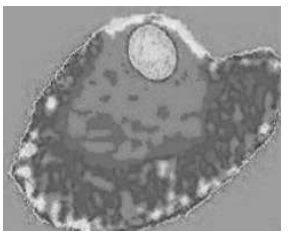

Watershed segmentation

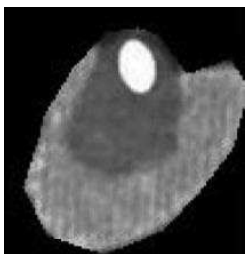

Right kidney

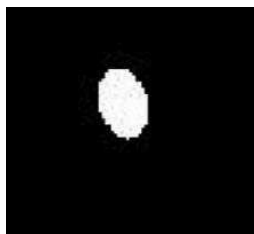

Stone

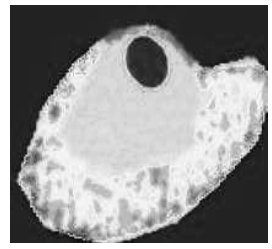

After coloring

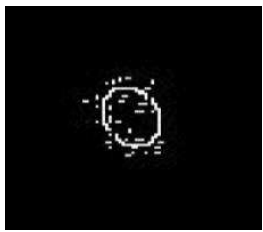

Boundary of Stone

Fig.(11): Shows the watershed segmentation with stones case for image No.1.

\section{Table (9)}

The statistical features for right kidney for the stone case.

\begin{tabular}{|c||c||c||c|}
\hline Image No.1 & $\begin{array}{c}\text { Standard } \\
\text { Deviation }\end{array}$ & Mean & $\begin{array}{c}\text { Local } \\
\text { Homogeneity }\end{array}$ \\
\hline \hline Right kidney & 61.2134 & 140.4874 & 0.9902 \\
\hline
\end{tabular}
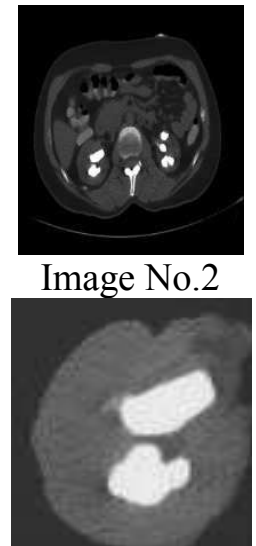

Watershed segmentation

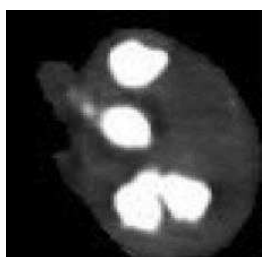

Left kidney

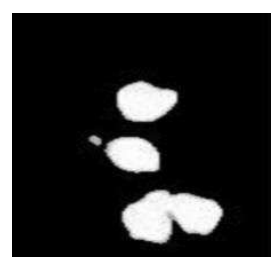

Stones

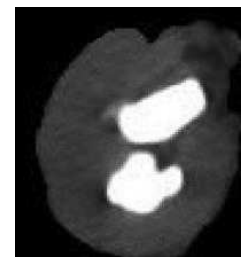

Right kidney

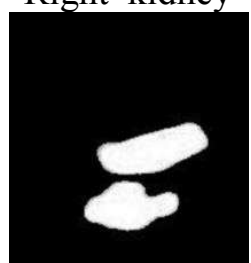

Stones

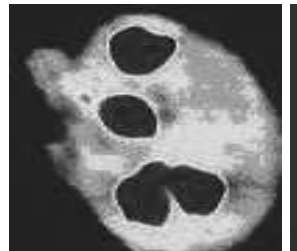

After coloring

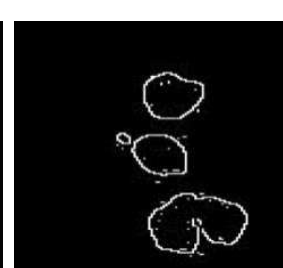

Boundary of Stones

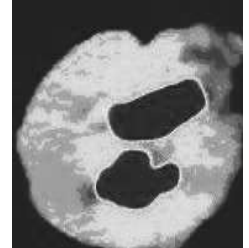

After coloring

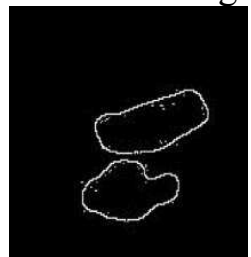

Boundary of Stones

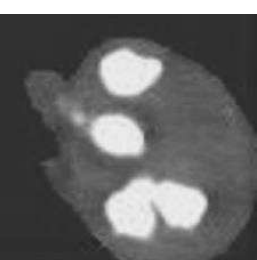

Watershed segmentation

Fig.(12): Shows the watershed segmentation with stones case for image No.2. 
Table (10)

The statistical features for right and left kidney for stone case.

\begin{tabular}{|c|c||c||c||}
\hline $\begin{array}{c}\text { Image } \\
\text { No.2 }\end{array}$ & $\begin{array}{c}\text { Standard } \\
\text { Deviation }\end{array}$ & Mean & $\begin{array}{c}\text { Local } \\
\text { Homogeneity }\end{array}$ \\
\hline \hline $\begin{array}{c}\text { Right } \\
\text { Kidney }\end{array}$ & 51.3542 & 104.83 & 0.9907 \\
\hline \hline $\begin{array}{c}\text { Left } \\
\text { Kidney }\end{array}$ & 54.3997 & 100.8215 & 0.9903 \\
\hline \hline
\end{tabular}

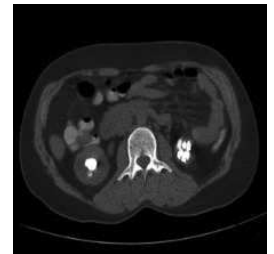

Image No.3

Watershed segmentation

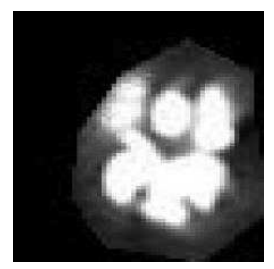

Left kidney

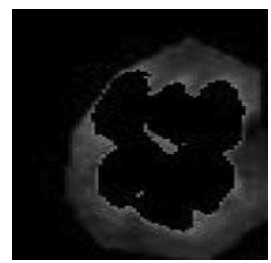

Healthy tissues

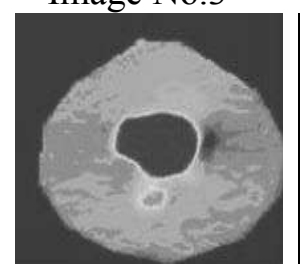

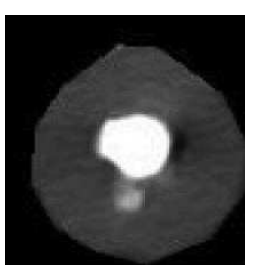

Right kidney

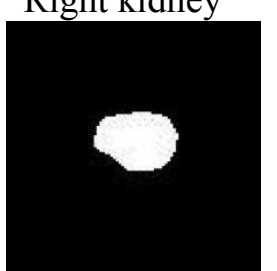

Stone

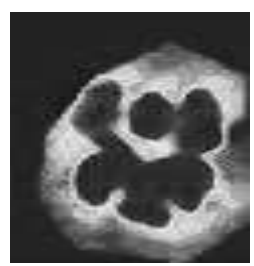

After coloring

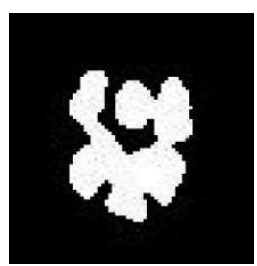

Stones

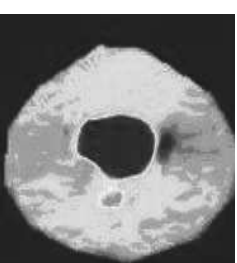

After coloring

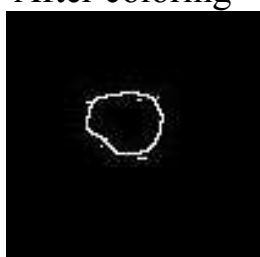

Boundary of stone

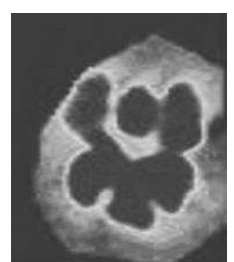

Watershed segmentation

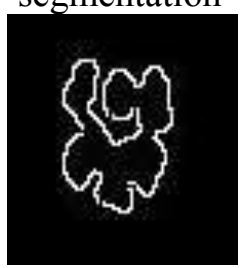

Boundary of stones
Fig.(13): Shows the watershed segmentation with stones case for image No.3.

Table (11)

The statistical features for right and left kidney for stone case.

\begin{tabular}{||c||c||c||c||}
\hline $\begin{array}{c}\text { Image } \\
\text { No.3 }\end{array}$ & $\begin{array}{c}\text { Standard } \\
\text { Deviation }\end{array}$ & Mean & $\begin{array}{c}\text { Local } \\
\text { Homogeneity }\end{array}$ \\
\hline \hline $\begin{array}{c}\text { Right } \\
\text { kidney }\end{array}$ & 48.9199 & 122.8317 & 0.9944 \\
\hline $\begin{array}{c}\text { Left } \\
\text { kidney }\end{array}$ & 44.7992 & 108.659 & 0.09964 \\
\hline
\end{tabular}

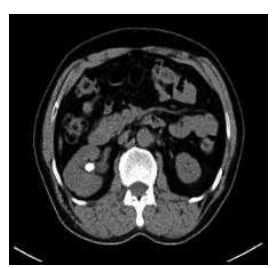

Image No.4

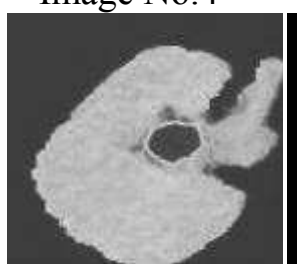

Watershed segmentation

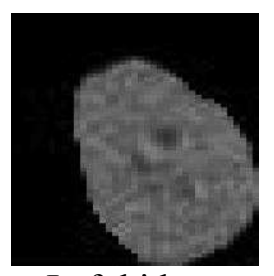

Left kidney

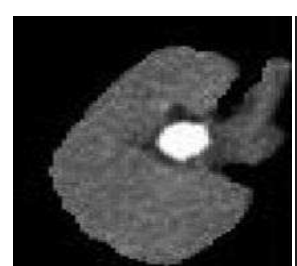

Right kidney

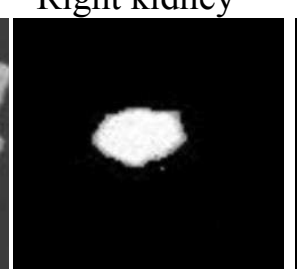

Stone

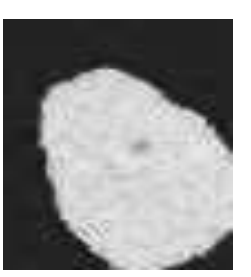

After coloring

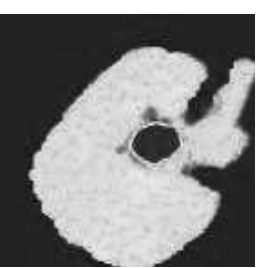

After coloring

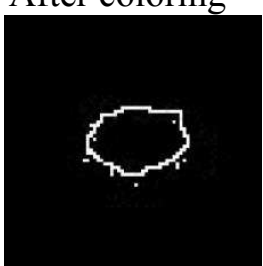

Boundary of stone

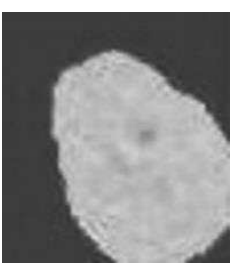

Watershed segmentation
Fig (14): Shows the watershed segmentation with stones case for image No.4.

\section{Table (12)}

The statistical features for right kidney for stone case.

\begin{tabular}{|c||c|c||c||}
\hline $\begin{array}{c}\text { Image } \\
\text { No.4 }\end{array}$ & $\begin{array}{c}\text { Standard } \\
\text { Deviation }\end{array}$ & Mean & $\begin{array}{c}\text { Local } \\
\text { Homogeneity }\end{array}$ \\
\hline \hline $\begin{array}{c}\text { Right } \\
\text { kidney }\end{array}$ & 60.0722 & 130.4249 & 0.9944 \\
\hline
\end{tabular}




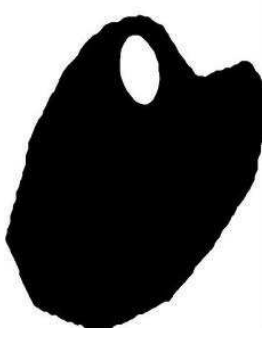

Image No.1 right side

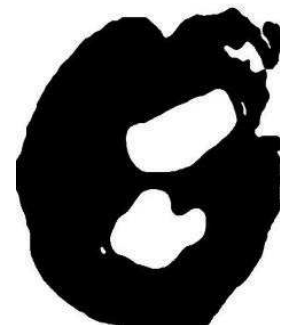

Image No.3 right side

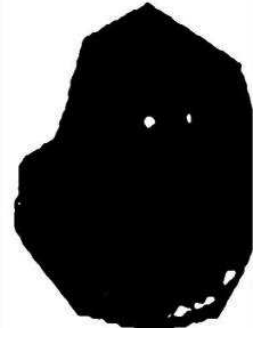

Image No.1 left side

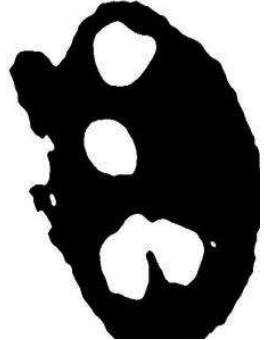

Image No.3 left side

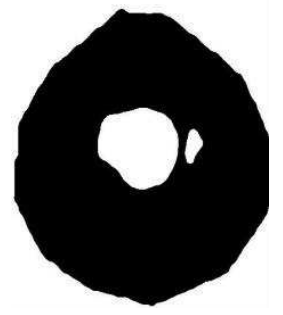

Image No.2 right side

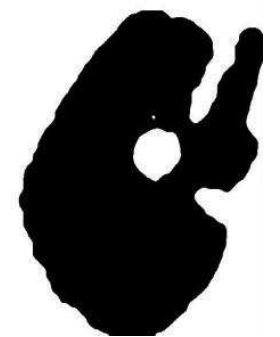

Image No.4 right side

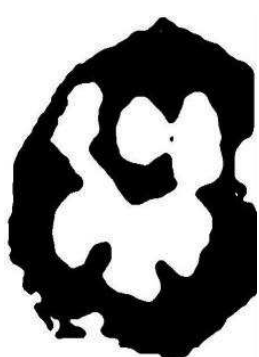

Image No.2 left side

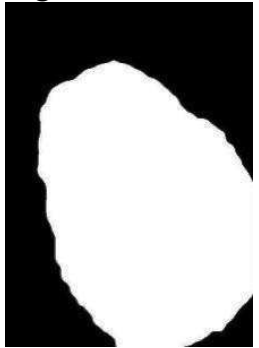

Image No.4 left side

Fig. (15): Shows the LBP images for Stone cases.

\section{Discussion}

A statistical feature are calculated for the cyst, fibrosis and the stone parts, the Local Binary Pattern which is a textural features extraction procedure are calculated for these cases, the images have been derived to the third derivation which the best result is obtained. The texture spectrum are also calculated to improve the textural unit. The geometrical feature represented by the area, circumference and the irregularity value represented the shape of the detected tumor. This value helps the physician to get the exact size and shape of tumor to give the patient the suitable amount of treatment Tables $(13,14$, $15,16,17,18)$ shows the irregularity values, Table (19) represented the average value for the three cases of the statistical features, Table (20) is the average value of the irregularity for the three cases, Table (21) is the textural feature accuracy for the three cases. The cyst has the greatest value in the statistical features and the most regular between the stone and fibrosis cases. The value of stander deviation is higher than the other cases (i-e) the variance is high and the dispersion around the mean is also high, the local homogeneity and mean for the cyst is high than the other cases. This can clearly see in Fig.(16).
Table (13)

The geometrical features value the Area, Perimeter and Irregularity Index of right kidney Cyst case.

\begin{tabular}{||c|c|c|c||}
\hline $\begin{array}{c}\text { Image } \\
\text { No. }\end{array}$ & $\begin{array}{c}\text { Area (A) } \\
\text { in pixels }\end{array}$ & $\begin{array}{c}\text { Perimeter } \\
\text { (P) in } \\
\text { pixel }\end{array}$ & $\begin{array}{c}\text { Irregularity } \\
\text { Index (I) }\end{array}$ \\
\hline \hline 1 & 13095 & 9675 & 0.0017570867 \\
\hline 2 & 29166 & 9366 & 0.004175978 \\
\hline 3 & 41880 & 13083 & 0.0030731349 \\
\hline 4 & 27183 & 13419 & 0.0018960366 \\
\hline
\end{tabular}

Table (14)

The geometrical features value the Area, Perimeter and Irregularity Index of left kidney Cyst case.

\begin{tabular}{|c||c||c||c||}
\hline $\begin{array}{c}\text { Image } \\
\text { No. }\end{array}$ & $\begin{array}{c}\text { Area (A) } \\
\text { in pixels }\end{array}$ & $\begin{array}{c}\text { Perimeter } \\
\text { (P) in } \\
\text { pixel }\end{array}$ & $\begin{array}{c}\text { Irregularity } \\
\text { Index (I) }\end{array}$ \\
\hline \hline 1 & 72336 & 33438 & 0.0008125752 \\
\hline \hline 2 & 44118 & 20352 & 0.0013378002 \\
\hline \hline 3 & 42069 & 15966 & 0.0020728104 \\
\hline 4 & 17220 & 9852 & 0.0022283016 \\
\hline
\end{tabular}


Table (15)

The geometrical features value the Area, Perimeter and Irregularity Index of right kidney Fibrosis case.

\begin{tabular}{||c||c|c||c||}
\hline $\begin{array}{c}\text { Image } \\
\text { No. }\end{array}$ & $\begin{array}{c}\text { Area } \\
(\mathbf{A}) \text { in } \\
\text { pixels }\end{array}$ & $\begin{array}{c}\text { Perimeter } \\
\text { (P) in } \\
\text { pixel }\end{array}$ & $\begin{array}{c}\text { Irregularity } \\
\text { Index (I) }\end{array}$ \\
\hline \hline 1 & 109581 & 78435 & 0.0002237201 \\
\hline 2 & 189057 & 123783 & 0.0001549745 \\
\hline \hline 3 & 257157 & 122163 & 0.0002164256 \\
\hline \hline 4 & 244023 & 74991 & 0.000545007 \\
\hline
\end{tabular}

Table (16)

The geometrical features value the Area, Perimeter and Irregularity Index of left kidney Fibrosis case.

\begin{tabular}{|c||c||c||c||}
\hline $\begin{array}{c}\text { Image } \\
\text { No. }\end{array}$ & $\begin{array}{c}\text { Area } \\
\text { (A) in } \\
\text { pixels }\end{array}$ & $\begin{array}{c}\text { Perimeter } \\
\text { (P) in } \\
\text { pixel }\end{array}$ & $\begin{array}{c}\text { Irregularity } \\
\text { Index (I) }\end{array}$ \\
\hline \hline 1 & 74541 & 55464 & 0.0003043424 \\
\hline 2 & 124263 & 88788 & 0.0001979807 \\
\hline 3 & 144270 & 86349 & 0.000243025 \\
\hline 4 & 134214 & 68448 & 0.0003598039 \\
\hline
\end{tabular}

Table (17)

The geometrical features value the Area, Perimeter and Irregularity Index of right kidney Stone case.

\begin{tabular}{|c||c|c|c|}
\hline $\begin{array}{c}\text { Image } \\
\text { No. }\end{array}$ & $\begin{array}{c}\text { Area } \\
\text { (A) in } \\
\text { pixels }\end{array}$ & $\begin{array}{c}\text { Perimeter } \\
\text { (P) in } \\
\text { pixel }\end{array}$ & $\begin{array}{c}\text { Irregularity } \\
\text { Index (I) }\end{array}$ \\
\hline \hline 1 & 9684 & 9882 & 0.0012455315 \\
\hline 2 & 30075 & 14061 & 0.0019105696 \\
\hline 3 & 3357 & 5088 & 0.0016287213 \\
\hline 4 & 4365 & 12222 & 0.0003670197 \\
\hline
\end{tabular}

Table (18)

The geometrical features value of the Area, Perimeter and Irregularity Index of left kidney Stone case.

\begin{tabular}{|c||c||c||c||}
\hline $\begin{array}{c}\text { Image } \\
\text { No. }\end{array}$ & $\begin{array}{c}\text { Area } \\
\text { (A) in } \\
\text { pixels }\end{array}$ & $\begin{array}{c}\text { Perimeter } \\
\text { (P) in } \\
\text { pixel }\end{array}$ & $\begin{array}{c}\text { Irregularity } \\
\text { Index (I) }\end{array}$ \\
\hline \hline 1 & $\backslash$ & $\backslash$ & $\backslash$ \\
\hline 2 & 19731 & 11628 & 0.001832857 \\
\hline 3 & 7068 & 12291 & 0.0005876405 \\
\hline \hline 4 & $\backslash$ & $\backslash$ & $\backslash$ \\
\hline
\end{tabular}

Table (19)

Shows the average value for the statistical features for the Cyst, Fibrosis and Stone cases.

\begin{tabular}{|c||c||c||c|}
\hline Cases & $\begin{array}{c}\text { Standard } \\
\text { Deviation }\end{array}$ & Mean & $\begin{array}{c}\text { Local } \\
\text { Homogeneity }\end{array}$ \\
\hline \hline Cyst & 55.1151 & 130.4256 & 0.9946 \\
\hline \hline Fibrosis. & 41.6132 & 85.2189 & 0.9747 \\
\hline \hline Stone & 53.45976667 & 118.0090833 & 0.992733333 \\
\hline
\end{tabular}

Table (20)

Shows the average value for the statistical features for the Cyst, Fibrosis and Stone cases.

\begin{tabular}{|c||c||}
\hline Cases & Irregularity \\
\hline \hline Cyst & 0.0021675 \\
\hline Fibrosis. & 0.0002796 \\
\hline \hline Stone & 0.00126205 \\
\hline
\end{tabular}


Table (21)

The textural Features Accuracy.

\begin{tabular}{|c|c|c|c|}
\hline \multicolumn{4}{|c|}{ Cyst } \\
\hline $\begin{array}{c}\text { Image } \\
\text { NO. }\end{array}$ & $\begin{array}{c}\text { Local } \\
\text { Binary } \\
\text { Pattern } \\
(\text { LBP)\% }\end{array}$ & $\begin{array}{l}\text { Textural } \\
\text { Spectrum } \\
\text { (TS) } \%\end{array}$ & $\begin{array}{c}\text { Local } \\
\text { Binary } \\
\text { Pattern } \\
\text { (LBP) and } \\
\text { Textural } \\
\text { Spectrum } \\
\text { (TS)\% } \\
\end{array}$ \\
\hline 1 (left) & 95.3 & 96.7 & 97.4 \\
\hline 2 (left) & 93.2 & 92.1 & 94.8 \\
\hline 3 (left) & 97.2 & 98.2 & 98.9 \\
\hline 4 (left) & 91.3 & 92.7 & 97.9 \\
\hline 1 (right) & 91.2 & 93.3 & 98.2 \\
\hline 2 (right) & 98.1 & 97.4 & 91.8 \\
\hline 3 (right) & 88.8 & 89.9 & 95.1 \\
\hline 4 (right) & 94.1 & 96.2 & 93.9 \\
\hline Average & $93.65 \%$ & $94.56 \%$ & $96 \%$ \\
\hline \multicolumn{4}{|c|}{ Fibrosis } \\
\hline $\begin{array}{c}\text { Image } \\
\text { NO. }\end{array}$ & $\begin{array}{c}\text { Local } \\
\text { Binary } \\
\text { Pattern } \\
(\text { LBP)\% }\end{array}$ & $\begin{array}{l}\text { Textural } \\
\text { Spectrum } \\
\text { (TS) } \%\end{array}$ & $\begin{array}{c}\text { Local } \\
\text { Binary } \\
\text { Pattern } \\
\text { (LBP) and } \\
\text { Textural } \\
\text { Spectrum } \\
\text { (TS)\% } \\
\end{array}$ \\
\hline 1 (left) & 97.1 & 99.2 & 88.9 \\
\hline 2 (left) & 96.4 & 97.8 & 98.4 \\
\hline 3 (left) & 90.8 & 91.9 & 99.4 \\
\hline 4 (left) & 81.9 & 87.8 & 98.1 \\
\hline 1 (right) & 95.7 & 96.8 & 96.7 \\
\hline 2 (right) & 88.7 & 89.7 & 92.8 \\
\hline 3 (right) & 78.8 & 86.8 & 89.9 \\
\hline 4 (right) & 89.9 & 91.2 & 92.2 \\
\hline Average & $89.91 \%$ & $92.65 \%$ & $94.55 \%$ \\
\hline \multicolumn{4}{|c|}{ Stone } \\
\hline $\begin{array}{c}\text { Image } \\
\text { NO. }\end{array}$ & $\begin{array}{c}\text { Local } \\
\text { Binary } \\
\text { Pattern } \\
(\text { LBP)\% }\end{array}$ & $\begin{array}{c}\text { Textural } \\
\text { Spectrum } \\
\text { (TS) } \%\end{array}$ & $\begin{array}{c}\text { Local } \\
\text { Binary } \\
\text { Pattern } \\
\text { (LBP) and } \\
\text { Textural } \\
\text { Spectrum } \\
\text { (TS)\% } \\
\end{array}$ \\
\hline 2 left & 92.9 & 92.7 & 93.5 \\
\hline 3left & 84.1 & 94.2 & 97.4 \\
\hline 1 right & 97.2 & 98.6 & 94.8 \\
\hline 2 right & 83.9 & 81.9 & 96.9 \\
\hline 3right & 88.9 & 89.6 & 96.8 \\
\hline 4 right & 83.4 & 91.2 & 91.8 \\
\hline Average & $88.4 \%$ & $91.36 \%$ & $95.2 \%$ \\
\hline
\end{tabular}

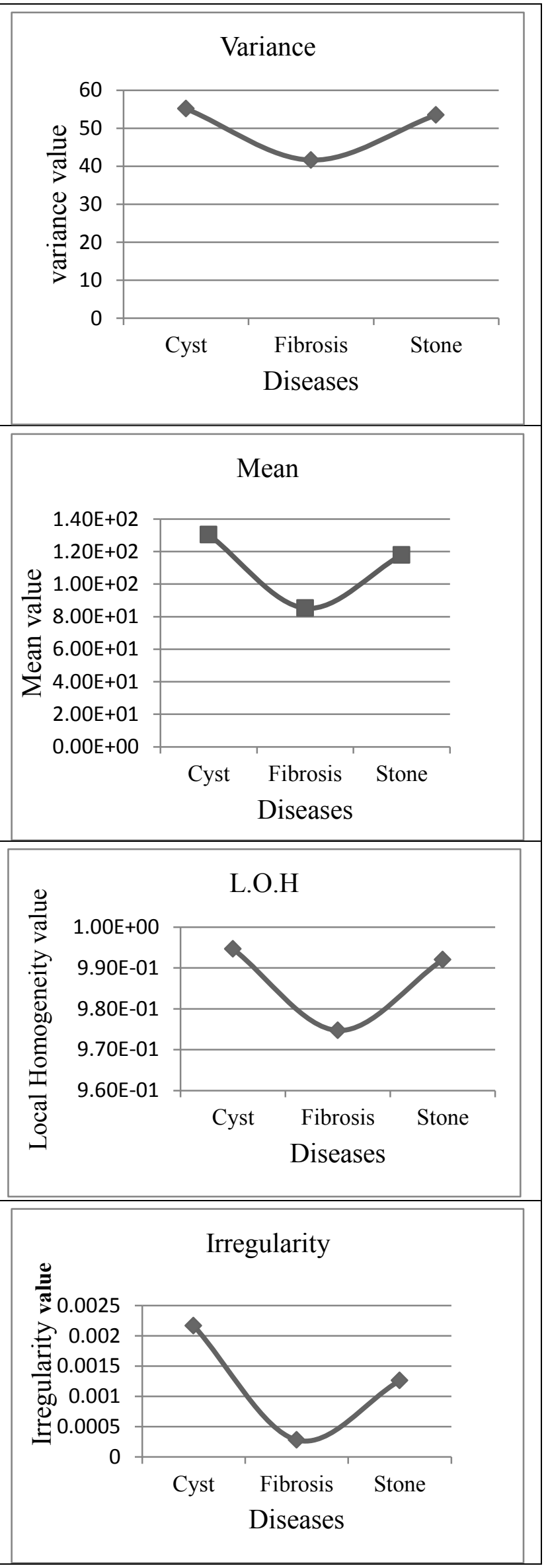

Fig.(16): The statistical and geometrical features for cyst, fibroses and stone. 


\section{Conclusion}

The cyst texture is the purest texture compared with the fibrosis and the stone cases, it local homogeneity is higher than the other, the stander deviation and mean is also, higher this means that the cyst has a Number of gray level more than the other cases. The irregularity value for the cyst is high compare with fibrosis and stone, its shape tend to be more regular and this can be shown in Fig.(5). Table (21) shows that the local binary pattern accuracy and textural spectrum is high for the cyst than for fibrosis and stone texture. The texture spectrum extracted the general features of the kidney, the combination between the (LBP) and (TS) gives good classification than (LBP) and (TS) individually and this cab clearly seen from the accuracy value. The accuracy value for the Cyst is $96 \%$ which is higher than Fibrosis and Stone.

\section{References}

[1] A. kaur, A. Verma, S. Derabassi, "The Marker-Based Watershed Segmentation- A Review", International Journal of Engineering and Innovative Technology (IJEIT) Volume 3, Issue 3, September. 2013.

[2] A. K. Jain, "Fundamentals of Digital Image Processing", Englewood Cliffs, Prentice Hall. 1989.

[3] B. Vijayalakshmi1 \& V. Subbiah Bharathi, "Texture Classification Using Combined Statistical Approach", International Journal of Applied Computing, 4(1), pp. 7-11. 2011.

[4] B. S. Manjunath, W. Y. Ma, "Texture Features for Browsing and Retrieval of Image Data", IEEE Transactions on Pattern Analysis and Machine, Volume 18, Issue 8, pp. 837-842. 1996.

[5] S. BEUCHER, "The Watershed Transformation Applied To Image Segmentation", Scanning microscopy. Supplement 6. Prentice Hall 2000.

[6] X. Zhang, L. Chen, L. Pan and L. Xiong, "Study on the Image Segmentation Based On ICA and Watershed Algorithm," Fifth International Conference on Intelligent Computation Technology and Automation, pp. 978-912, IEEE. 2012.
[7] R. C.Gonzalez and R.E.Woods, (Digital Image Processing), Third Edition. Pearson Prentice Hall 2008.

[8] S. Mazhir, "Studying the Effect of Cold Plasma on Living Tissues Using Images Texture analysis", Diyala Journal for Pure Science. vol. 13. No.2. 2017.

[9] Ch. Chuan, "Thyroid Nodule Segmentation and Component Analysis in Ultrasound Images" Proceeding: Asia-Pacific Signal and Information Processing Association. 2009.

[10] U S N Raju, A Sridhar Kumar, B Mahesh, B Eswara Reddy, "Texture Classification With High Order Local Pattern Descriptor: Local Derivative Patter", GJCST, Vol.10, Issue 8 Ver. 1, pp72. 2010.

[11] A. H.Ali, E. M.Hadi, and S. N. Mazhir, "Diagnosis of liver Tumor from CT Images Using Unsupervised Classification with Geometrical and Statistical features", IJACSSE, Vol.5, Issue3. 2015. 